\title{
Orientational dependence of optically detected magnetic resonance signals in laser-driven atomic magnetometers
}

\author{
Simone Colombo ${ }^{1}$ (D) Vladimir Dolgovskiy ${ }^{1}$ Theo Scholtes $^{1} \cdot$ Zoran D. Grujić $^{1} \cdot$ \\ Victor Lebedev ${ }^{1} \cdot$ Antoine Weis $^{1}$
}

Received: 2 September 2016 / Accepted: 28 November 2016 / Published online: 29 December 2016

(c) The Author(s) 2016. This article is published with open access at Springerlink.com

\begin{abstract}
We have investigated the dependence of lock-indemodulated $M_{x}$-magnetometer signals on the orientation of the static magnetic field $\mathbf{B}_{0}$ of interest. Magnetic resonance spectra for 2400 discrete orientations of $\mathbf{B}_{0}$ covering a $4 \pi$ solid angle have been recorded by a PC-controlled steering and data acquisition system. Off-line fits by previously derived lineshape functions allow us to extract the relevant resonance parameters (shape, amplitude, width, and phase) and to represent their dependence on the orientation of $\mathbf{B}_{0}$ with respect to the laser beam propagation direction. We have performed this study for two distinct $M_{x}$ -magnetometer configurations, in which the rf-field is either parallel or perpendicular to the light propagation direction. The results confirm well the algebraic theoretical model functions. We suggest that small discrepancies are related to hitherto uninvestigated atomic alignment contributions.
\end{abstract}

\section{Introduction}

Optically pumped atomic magnetometers, also known as optical magnetometers (OM), are based on resonant magneto-optical effects in atomic (usually alkali-metal) vapors [1]. We refer the reader to the comprehensive overview of various OM methods and their applications in Ref. [2]. Magnetometers based on optically detected magnetic

This article is part of the topical collection "Enlightening the World with the Laser" - Honoring T. W. Hänsch guest edited by Tilman Esslinger, Nathalie Picqué, and Thomas Udem.

Simone Colombo

simone.colombo@unifr.ch

1 Physics Department, University of Fribourg, Chemin du Musée 3, CH-1700 Fribourg, Switzerland resonance (ODMR) have the longest history in the field of atomic magnetometry, and the so-called $M_{x}$-magnetometer using a single light beam has proven to be a highly sensitive and robust device. The theoretical modeling of the signals generated by ODMR-based magnetometers is addressed in great detail in a recently published textbook (Chapter 13 in Ref. [3]).

ODMR magnetometers infer the modulus $B_{0}=\omega_{0} / \gamma_{F}$ of the magnetic field vector $\mathbf{B}_{0}$ from the (driven) Larmor precession frequency $\omega_{0}$ of an atomic vapor's magnetization, where $\gamma_{\mathrm{F}}$ is the gyromagnetic ratio of the used atom $\left(\gamma_{F} / 2 \pi \approx 3.5 \mathrm{~Hz} / \mathrm{nT}\right.$ for $\left.{ }^{133} \mathrm{Cs}\right)$. The precession is driven by a much weaker additionally applied oscillating field $\mathbf{B}_{1}(t)$, called the 'rf-field'. In the standard $M_{x}$-magnetometer, a single circularly polarized light beam whose frequency is resonant with an atomic transition is used both to create the medium's spin polarization by optical pumping [4] and to read out the spin precession signal.

The magnetometric sensitivity of an OM, i.e., the smallest magnetic field change that the device can detect (in a given bandwidth), depends on many parameters, such as the light intensity, the atomic number density, the size of the atomic sample, the spin coherence time, and the amplitude of the rf-field. Moreover, the sensitivity critically depends on the relative orientations of the light propagation direction $\hat{k}$, the rf-field $\hat{\mathbf{B}}_{1}$, and the field of interest $\hat{\mathbf{B}}_{0}$. The latter dependencies imply that there are, on the one hand, orientation(s) that optimize the device's sensitivity, and, on the other hand, orientations (so-called dead-zones) for which the sensitivity vanishes. The quantitative understanding of these dependencies is crucial when designing a magnetometer, be it for a laboratory application in which the orientation $\hat{\mathbf{B}}_{0}$ of $\mathbf{B}_{0}$ is mostly known a priori, or for field applications where the knowledge of the dead-zones is of great importance. 
The problem of the OM sensitivity's orientation dependence is closely related to the so-called 'heading error' that has already been addressed in the very early accounts on optically pumped atomic magnetometers [5]. Several attempts have been made to overcome those fundamental effects and realize dead-zone-free OM [6-10].

The object of the present paper is an experimental verification of the theoretically predicted [3] orientation dependencies of lock-in-detected signals in different $M_{x}$ -magnetometers of two distinct geometrical configurations, viz., rf-field either parallel or perpendicular to the light's $\mathbf{k}$ -vector. For this, we have developed a computer-controlled experimental setup allowing the rotation of a static magnetic field vector of constant modulus over the full $4 \pi$ solid angle. We record magnetic resonance spectra at 2400 discrete $\left(\theta_{B}, \phi_{B}\right)$ orientations of the field, and off-line analysis permits then three-dimensional representations of the results.

\section{Experimental setup}

The experimental setup (Fig. 1) is mounted inside of a cubic five-layer $\mu$-metal shield (produced by Sekels $\mathrm{GmbH}$ ) with inner dimensions of $\sim 50^{3} \mathrm{~cm}^{3}$.

The central part of the magnetometer is a spherical (30 mm diameter) Pyrex cell with paraffin-coated inner surface which is connected by a capillary to a reservoir stem containing a droplet of solid cesium producing a

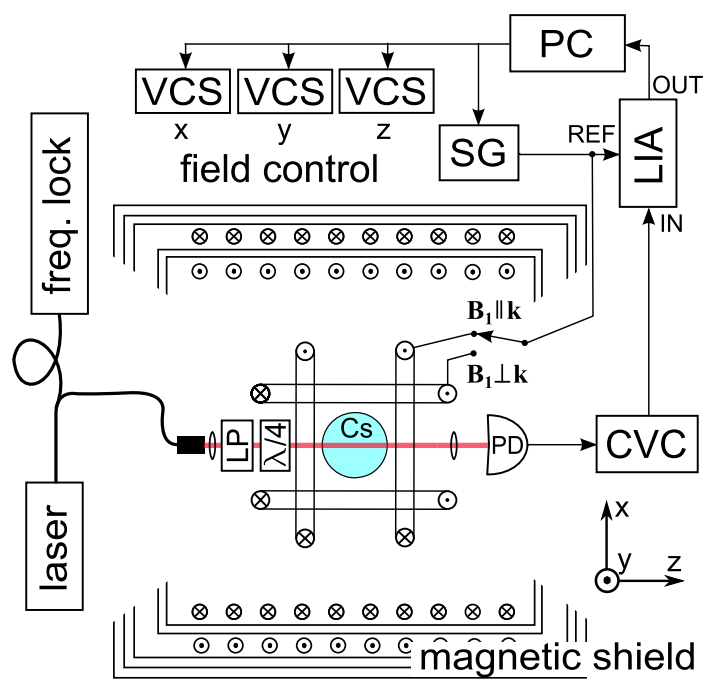

Fig. 1 Schematic of the experimental setup. The coils producing the $\mathbf{B}_{0}$ field's $x, y$, and $z$ components are wound around the two innermost $\mu$-metal layers. Two of the four coils producing $B_{z}$ are shown as illustration. $V C S$ voltage-controlled current source, $S G$ signal generator, it LIA lock-in amplifier, $L P$ linear polarizer, $P D$ photodiode, $C V C$ current-to-voltage converter, $P C$ computer running control and data acquisition code room-temperature saturated atomic vapor [11]. Laser light is guided to the setup by a multimode fiber which effectively scrambles the light polarization. The out-coupled ( $\approx 2 \mathrm{~mm}$ diameter) light beam's polarization is made circular using a linear polarizer and a quarter-wave plate. We use a polarimeter (Thorlabs, model PAX5710IR1-T) for the precision control of the light's polarization (cf. Sect. 7.1).

The frequency of the extended cavity diode laser (Toptica, model DL100 pro) is actively stabilized to the center of the $F=4 \rightarrow F^{\prime}=3$ hyperfine transition of the Cs $D_{1}$ line $(\lambda=894.6 \mathrm{~nm})$ by means of a separate saturation-absorption spectroscopy unit. The power of the laser beam is kept constant by an active stabilization circuit using an intensity modulator (Jenoptik, model AM894) driven by a slow PI controller with a $10 \mathrm{~Hz}$ cutoff frequency. The power of the transmitted light beam is detected by a photodiode whose photocurrent is amplified by a current-to-voltage converter (Femto, model DLPCA-200, $10^{6} \mathrm{~V} / \mathrm{A}$ gain, $200 \mathrm{kHz}$ bandwidth) and fed to a lock-in detector (Zurich Instruments, model HF2LI).

Magnetic resonance spectra are recorded by automated frequency sweeps of the rf-coil current produced by the built-in oscillator of the lock-in amplifier. The frequency dependence of both the in-phase and quadrature signals obtained by phase-sensitive demodulation of the photodiode signal is stored for off-line processing.

The amplifier's bandwidth and the finite inductivity of the rf-coil imply that the photocurrent's Fourier component of interest (oscillating at $\sim 35 \mathrm{kHz}$ ) is phase-shifted by a (frequency dependent) value $\varphi$ of $\approx 20^{\circ}$ with respect to the coil-driving voltage.

Data taking is fully computer-controlled by a dedicated LabView code allowing control of the rf-field amplitude and the three components of the offset field $\mathbf{B}_{\mathbf{0}}$.

\subsection{Magnetic field control and calibration}

The static magnetic field inside the shield is produced by a triaxial coil system wound onto the two innermost $\mu$-metal layers. The coils (resistance $4.5 \Omega$ ) are driven by voltages from three programmable arbitrary waveform generators (Agilent, model 33500B) via $50 \Omega$ series resistors. After demagnetization, we measure the remnant field components in the shield using level-crossing (Hanle) resonances as described in Refs. [12, 13]. We nullify these components - that are typically below 70nT-and calibrate the field producing coils in the following way: For calibrating the $B_{z}$ coil, we use an $M_{z}$ geometry, in which we scan $B_{z}$ from negative to positive values, while irradiating the atoms with a $1.5 \mathrm{kHz}$ rf-field. From the positions of the two magnetic resonances observed in this configuration, we infer both the residual field $\delta B_{z}$ and the coil calibration constant $k_{z}$. The $B_{x}$ and $B_{y}$ coils are calibrated using a $10 \mu \mathrm{T}$ 

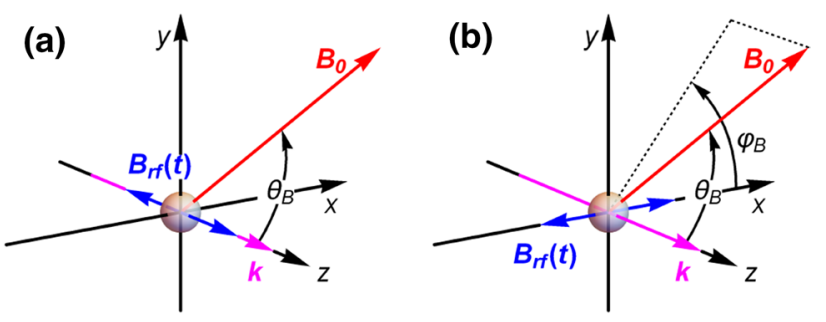

Fig. 2 Definition of the orientation angles $\theta_{B}$ and $\phi_{B}$ of the magnetic field in the geometries $\mathbf{B}_{1} \| \mathbf{k}(\mathbf{a})$ and $\mathbf{B}_{1} \perp \mathbf{k}(\mathbf{b})$

offset field $\left(0, B_{0 y}, B_{0 z}\right)$ direction. We measure changes of the corresponding Larmor frequency

$f_{0}=\gamma_{F} \sqrt{\left(k_{x} I_{x}\right)^{2}+\left(B_{0 y}+k_{y} I_{y}\right)^{2}+B_{0 z}^{2}}$

when powering the $x$ - and $y$-coils individually. Fits allow then to infer $k_{x}$ and $k_{y}$ as well as the residual fields $\delta B_{x}$ and $\delta B_{y}$.

We have observed current scan direction-related (sub$\%$ ) differences of the $k_{x}$ and $k_{y}$ constants. These differences may be attributed to the nonlinear ferromagnetic response of the $\mu$-metal onto which the coils are wound. The calibration constant of a given coil may thus be affected by the orientation and the magnitude of the offset field. Based on this, we believe that we control the field orientation at the $\lesssim 1 \%$ level.

Using this calibration, the generators are programmed such as to vary the $\theta_{B^{-}}$and $\phi_{B^{-}}$orientations of the magnetic field vector $\mathbf{B}_{\mathbf{0}}$ in a step-wise manner, while keeping the field modulus $B_{0}$ (nominally) constant. The field is varied on a full sphere evolving from the north pole $\left(0,0, B_{0}\right)$ to the south pole $\left(0,0,-B_{0}\right)$ in the coordinates of Fig. 1.

We record magnetic resonance spectra for typically 2400 pairs of discrete values of the field orientation angles (Fig. 2). The duration of each spectrum scan is $\approx 23 \mathrm{~s}$, so that a complete full-sphere orientation scan (' $\theta-\phi$-scan') takes $\approx 15 \mathrm{~h}$.

\section{Theory of the $M_{x}$-magnetometer}

The so-called $M_{x}$-magnetometer relies on reading out (by optical means) the frequency at which an atomic medium's spin polarization precesses around a static magnetic field $\mathbf{B}_{\mathbf{0}}$. The precession is coherently driven by a magnetic field (rf-field) of small amplitude $B_{1} \ll B_{0}$ that oscillates at a frequency $\omega_{\text {rf }}$ close to the Larmor frequency $\omega_{0}=\gamma_{F} B_{0}$. The ensuing phase-synchronized precession of all individual spins leads to a modulation of the transmitted light power at the frequency $\omega_{\mathrm{rf}}$. The amplitude (and phase) of the induced power modulation depends in a resonant manner on the detuning $\delta \omega \equiv \omega_{\mathrm{rf}}-\omega_{0}$ of the rf frequency from the Larmor frequency. Weis, Grujić ,and Bison have presented an exhaustive discussion of the mathematical expressions for the observed lineshapes in magnetic resonance-based atomic magnetometers in a recently published textbook [3].

The simplest $M_{x}$-magnetometer implementation uses a single circularly polarized laser beam (in resonance with an atomic transition) that serves for both the creation of the spin polarization and for the readout of the coherently driven spin precession. The laser power transmitted through the atomic vapor, which is assumed to be optically thin $(\kappa L \ll 1)$, is given by

$P=P_{0} \exp [-\kappa L] \approx P_{0}(1-\kappa L)$,

where $P_{0}$ is the incident light power and $L$ the length of the traversed vapor column. The absorption coefficient $\kappa$ for circularly polarized light depends on the medium's spin polarization and can be expressed as

$\kappa=\kappa_{0}^{\mathrm{unpol}}\left[1-\alpha_{F, F^{\prime}}^{(1)} S_{z}-\alpha_{F, F^{\prime}}^{(2)} A_{z z}\right]$,

where the coefficients $\alpha_{F, F^{\prime}}^{(K)}$ are transition-specific constants, and where $\kappa_{0}^{\text {unpol }}$ is the absorption coefficient of the unpolarized medium, $S_{z}$ and $A_{z z}$ being the medium's longitudinal spin orientation (vector polarization) and alignment (tensor polarization), respectively. Since alignment-related contributions on the $4 \rightarrow 3$ transition studied here are small [14], we will neglect in the following the last term in Eq. (3). The subscript $z$ refers to the quantization axis, chosen along the light propagation direction $\mathbf{k}$. In that frame, the longitudinal orientation can be expressed in terms of the sublevel populations $p_{m}$ as

$S_{z} \propto \sum_{m=-F}^{F} p_{m} m$,

where $m$ is the magnetic quantum number (eigenvalue of $F_{z}$ ). In what follows $S_{0}$ is the value of $S_{z}$ achieved by optical pumping in a (polarization-stabilizing) magnetic field $\mathbf{B}_{\mathbf{0}}$ oriented along $\mathbf{k}$.

The joint action of the torques exerted by the offset magnetic field $\mathbf{B}_{0}$ and the oscillating rf-field $\mathbf{B}_{\mathrm{rf}}(t)=B_{1} \hat{\mathbf{B}}_{1} \sin \omega_{\mathrm{rf}} t$ on the spin polarization causes a modulation of $S_{z}(t)$, and hence a time-dependent modulation

$$
\begin{aligned}
\delta P(t) & =P_{0} \kappa_{0}^{\mathrm{unpol}} L \alpha_{F, F^{\prime}}^{(1)} S_{z}(t) \\
& \equiv P_{\mathrm{R}}(\delta \omega) \sin \left[\omega_{\mathrm{rf}} t+\varphi(\delta \omega)\right] \\
& \equiv P_{\mathrm{IP}}(\delta \omega) \sin \omega_{\mathrm{rf}} t+P_{\mathrm{QU}}(\delta \omega) \cos \omega_{\mathrm{rf}}
\end{aligned}
$$


of the transmitted laser power, where $\delta \omega=\omega_{\mathrm{rf}}-\omega_{0}$ is the rf frequency detuning, and $P_{\mathrm{IP}}=P_{\mathrm{R}} \cos \varphi$ the in-phase and $P_{\mathrm{QU}}=P_{\mathrm{R}} \sin \varphi$ the quadrature amplitude, respectively. As shown in [3], the power modulation is phase-shifted with respect to the rf-field oscillation by

$\varphi(\delta \omega)=\varphi\left(\delta \omega ; \hat{\mathbf{B}}_{1}, \hat{\mathbf{B}}_{0}\right)=\varphi^{(0)}\left(\hat{\mathbf{B}}_{1}, \hat{\mathbf{B}}_{0}\right)-\arctan \frac{\delta \omega}{\gamma}$,

where the on-resonance phase shift $\varphi^{(0)}=\varphi(\delta \omega=0)$ depends both on the orientation, $\hat{\mathbf{B}}_{1}$, of the rf-field and on the orientation, $\hat{\mathbf{B}}_{0}$, of the magnetic field of interest.

We choose $\hat{z}=\hat{\mathbf{k}}$ as polar axis of a spherical coordinates system, so that the magnetic field orientation is determined by the polar and the azimuthal angles, $\theta_{B}$ and $\phi_{B}$, respectively. In this coordinate frame, the amplitude of the detected light power oscillation (' $R$ '-signal) is given by

$P_{R}(\delta \omega)=\widetilde{P} \frac{\sqrt{\gamma^{2}+\delta \omega^{2}}}{\delta \omega^{2}+\gamma^{2}+\widetilde{\Omega}_{i}^{2}} \widetilde{\Omega}_{i}\left|\sin \theta_{B} \cos \theta_{B}\right|$,

where $\gamma$ is the spin relaxation rate, which is assumed to be isotropic (setting $\gamma=\gamma_{1}=\gamma_{2}$ ), and where the signal calibration factor is given by $\widetilde{P}=S_{0} P_{0} \kappa_{0}^{\text {unpol }} L \alpha_{F, F^{\prime}}^{(1)}$.

As discussed in Ref. [3], all $M_{x}$-magnetometer signals can be represented in the form of Eqs. (8) and (9). We therefore refer to these expressions as a "universal representation' of the $M_{x}$-magnetometer signal, whose spectral dependence (but not its absolute value) is independent of the orientation of the rf-field. The interest of writing the phase signal in the universal form lies in the fact thatafter electronic subtraction of the offset phase $\varphi^{(0)}$ depending on $\hat{\mathbf{B}}_{0}$ and $\hat{\mathbf{B}}_{1}$ - the frequency dependence of the phase has a pure arctan dependence on the detuning with $\varphi=0$ on resonance and a negative slope $\mathrm{d} \varphi / \mathrm{d} \delta \omega$.

We define the Rabi frequency associated with the rf-field as $\Omega=\frac{1}{2} \gamma_{\mathrm{F}} \mathrm{B}_{1}$. Since the component of $\mathbf{B}_{1}$ along $\mathbf{B}_{0}$ does not induce magnetic resonance transitions, we have introduced in (9) an 'effective' Rabi frequency $\widetilde{\Omega}$ that is associated with the component of $\mathbf{B}_{1}$ that is orthogonal to $\mathbf{B}_{0}$, viz.,

$\widetilde{\Omega}_{\hat{\mathbf{B}}_{1}}\left(\hat{\mathbf{B}}_{0}\right)=\Omega\left|\hat{\mathbf{B}}_{1}-\left(\hat{\mathbf{B}}_{1} \cdot \hat{\mathbf{B}}_{0}\right) \hat{\mathbf{B}}_{0}\right|$.

Below we will describe experiments carried out with $M_{x}$ -magnetometers having two distinct orientations of the rf-field with respect to the light propagation direction, as shown in Fig. 2:

- The $\mathbf{B}_{1} \| \mathbf{k}$ geometry shown in Fig. 2a has a cylindrical symmetry around the $\mathbf{k}$-vector, so that one expects the magnetometer signals only to depend on the polar angle $\theta_{B}$. One easily sees that in this case the effective Rabi frequency is given by
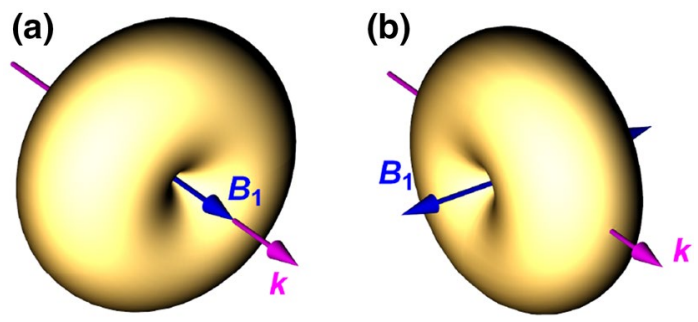

Fig. 3 Anticipated angular dependencies of the effective Rabi frequencies $\widetilde{\Omega}_{\hat{\mathbf{B}}_{1}}$ for the $\mathbf{B}_{1} \| \mathbf{k}(\mathbf{a})$ and $\mathbf{B}_{1} \perp \mathbf{k}(\mathbf{b})$ geometries

$\widetilde{\Omega}_{\|}\left(\theta_{B}, \phi_{B}\right)=\Omega\left|\sin \theta_{B}\right|$,

while the on-resonance phase is given by

$\varphi_{\|}^{(0)} \equiv \varphi^{(0)}\left(\hat{z},\left\{\theta_{B}, \phi_{B}\right\}\right)=-\frac{\pi}{2} \operatorname{sign}\left(\cos \theta_{B}\right)$.

We note that the offset phase is erroneously given in Ref. [3] (the referred work appeared while preparing the present text) as $\varphi_{\|}^{(0)}=0$, while the above result is the correct expression using the 4-quadrant definition of the arctan function for inferring the phase.

- The $\mathbf{B}_{1} \perp \mathbf{k}$ geometry shown in Fig. $2 b$ is no longer rotationally invariant around $\mathbf{k}$, and it is shown in [3] that in that case one has

$$
\begin{aligned}
& \widetilde{\Omega}_{\perp}\left(\theta_{B}, \phi_{B}\right)=\Omega \sqrt{\cos ^{2} \theta_{B}+\sin ^{2} \theta_{B} \sin ^{2} \phi_{B}} \\
& \begin{aligned}
\varphi_{\perp}^{(0)} & =\varphi^{(0)}\left(\hat{x},\left\{\theta_{B}, \phi_{B}\right\}\right) \\
& =\arctan \left(\cos \theta_{B} \cot \phi_{B}\right) .
\end{aligned}
\end{aligned}
$$

Figure 3 represents the orientation dependencies given by Eqs. (11) and (13).

The on-resonance amplitudes of the $R$-signals for the two cases (distinguished by the direction $\hat{\mathbf{B}}_{1}$ ) are then given by

$P_{R}^{\hat{\mathbf{B}}_{1}}(0)=\tilde{P} \frac{\gamma \widetilde{\Omega}_{\hat{\mathbf{B}}_{1}}\left(\theta_{B}, \phi_{B}\right)}{\gamma^{2}+\widetilde{\Omega}_{\hat{\mathbf{B}}_{1}}^{2}\left(\theta_{B}, \phi_{B}\right)}\left|\sin \theta_{B} \cos \theta_{B}\right|$.

\section{Data recording}

After the careful calibration of the coils generating the $x$-, $y$ - and $z$-components of the field vector $\mathbf{B}_{0}$, we orient the latter in a systematic manner along 2400 discrete directions, while keeping the field modulus $B_{0}$ constant. For this, we feed suitable voltages to the three coils via three series resistors. The voltages are generated by computercontrolled programmable signal generators. A full $4 \pi$ scan is achieved by varying $\theta_{B}$ from $0^{\circ}$ to $180^{\circ}$ in 40 equidistant 
(a)

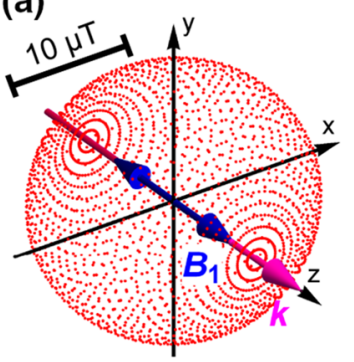

(b)

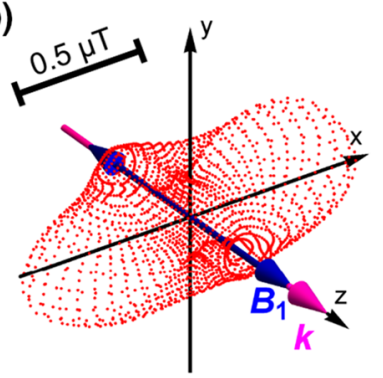

(a)

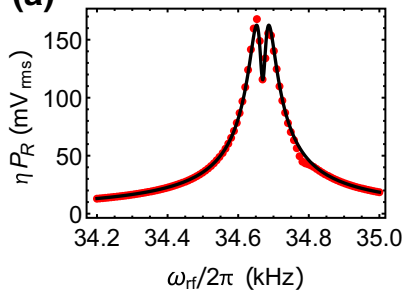

(b)

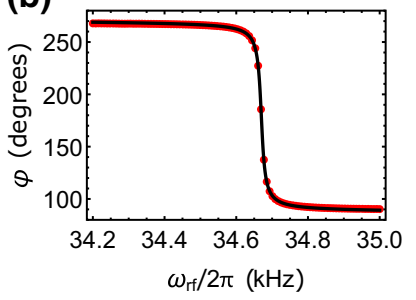

Fig. 5 Typical rf frequency dependence of the amplitude (a) and phase (b) signals of the demodulated detected laser power. Data shown as red points together with fitted functions [Eqs. (17), (16)] shown as solid lines. Data taken with the magnetometer operated in the transverse $\left(\mathbf{B}_{1} \perp \mathbf{k}\right)$ geometry with $\hat{\mathbf{B}}_{0}$ oriented along $\theta_{B}=45.67^{\circ}$, $\phi_{B}=90^{\circ}$ and an effective Rabi frequency of $\widetilde{\Omega}=2.0 \gamma$

steps. For each value of $\theta_{B}$, we vary the azimuthal angle $\phi_{B}$ from $0^{\circ}$ to $360^{\circ}$ in 60 equidistant steps.

The field modulus $B_{0}$ is chosen to be $\sim 10 \mu \mathrm{T}$, which corresponds to a Larmor frequency $f_{0}=\frac{\omega_{0}}{2 \pi}$ of $\sim 35 \mathrm{kHz}$. For each field orientation $\left(\theta_{B}, \phi_{B}\right)$, we scan the rf frequency $\omega_{\text {rf }}$ across a range of $\pm 400 \mathrm{~Hz}$ (in $8 \mathrm{~Hz}$ steps) around the Larmor frequency $\omega_{0}$. To ensure that the resonance line is always well centered in the scan range, we do a coarse (automated) determination of the line center after each recording in order to define the start- and stop-frequencies for the scan at the subsequent $\theta_{B}-\phi_{B}$ orientation.

Figure 4a shows the spatial distribution of the Larmor frequencies extracted from the off-line analysis of the data discussed in the next section. The average frequency of the displayed points is $36.0(3) \mathrm{kHz}$. However, we find that during the angular scans the Larmor frequency varies in a systematic manner over a range of $\pm 1 \mathrm{kHz}$ with respect to that average. This variation has an rms value of $0.9 \%$ (compatible with the $1 \%$ estimated uncertainty of the calibration constants $k_{i}$ ) and is represented in Fig. 4b, where we show the data of Fig. 4a after subtraction of $35.4 \mathrm{kHz}$.

\section{Data analysis}

The digital lock-in amplifier records and stores the in-phase and quadrature signals (Cartesian coordinates) as well as the $R$-signal and phase (polar coordinates). We have discovered a synchronous (but phase-shifted) photodiode signal (oscillating at the rf frequency) that is present even in absence of light. We assign this signal to inductive pickup of the rf-field by the photodiode's electrical circuit. We have recorded this signal with the laser beam blocked and subtract its in-phase and quadrature components from the corresponding magnetometer signals in the off-line analysis. After this correction we calculate the amplitude $\left(P_{R}\right)$ and phase $(\varphi)$ signals from the in-phase and quadrature (a)
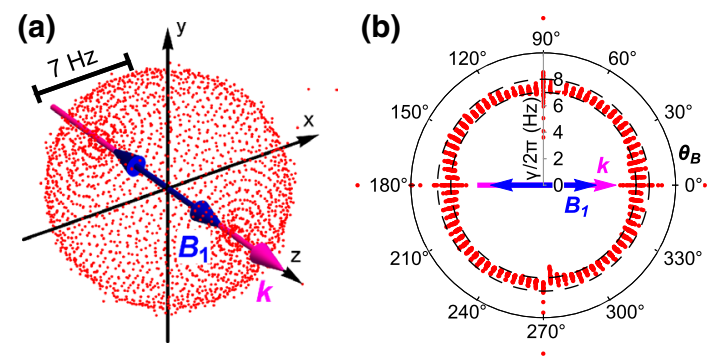

Fig. 6 a Angular distribution of the relaxation rates (magnetic resonance linewidths) $\gamma / 2 \pi$ as inferred from the parameters $\gamma_{\text {fit }}$ obtained by fitting the experimental $\varphi(\delta \omega)$ curves with Eq. (16); b: The same data as a function of $\theta_{B}$, with data for all $\phi_{B}$ values superposed

signals. From the phase signal we subtract the frequencydependent phase shift of $\approx 20^{\circ}$ mentioned in Sect. 2. Typical results are shown in Fig. 5.

We then fit the theoretical expressions for $\varphi(\delta \omega)$ and $P_{R}(\delta \omega)$ given by Eqs. (8) and (9), respectively, to the data. We note that the experimental phase signal is determined by the ratio of the quadrature and the in-phase signals, so that $\varphi_{\exp }(\delta \omega)$ does not depend on the common prefactor of those signals. We therefore first fit the function

$\varphi_{\text {fit }}\left(\omega_{\text {rf }} ; \omega_{\text {fit }}, \gamma_{\text {fit }}, \varphi_{\text {fit }}^{(0)}\right)=\varphi_{\text {fit }}^{(0)}-\arctan \frac{\omega_{\text {rf }}-\omega_{\text {fit }}}{\gamma_{\text {fit }}}$

to the experimental phase signals $\varphi_{\exp }(\delta \omega)$. Besides 'horizontal' $\left(\omega_{\text {fit }}\right)$ and 'vertical' $\left(\varphi_{\text {fit }}^{(0)}\right)$ offsets, the shape of the phase signals is fully determined by the relaxation rates $\gamma_{\text {fit }}$. The fits yield the resonance (Larmor) frequency, $\omega_{\mathrm{fit}}$, the (light power broadened) linewidth, $\gamma_{\text {fit }}$, as well as the offset phase, $\varphi_{\text {fit }}^{(0)}$ for each field orientation. We note that the resonance frequencies $\omega_{\text {fit }}$ determined from such fits are the values used in Figs. 4.a,b.

Figure $6 a$ shows the angular dependence of the fitted relaxation rates on the field orientation. In Fig. $6 \mathrm{~b}$ we represent a superposition of the 30 cuts through the sphere of Fig. $6 \mathrm{a}$, one for each of the $30 \phi_{B}$ angles. Each given cut 
thus contains $80 \gamma\left(\theta_{B}\right)$ points. The average value of all data is $\gamma /(2 \pi)=7.3(4) \mathrm{Hz}$.

The slight deviation from spherical symmetry observed near the dead-zones occurring at $\theta_{B}=n \pi / 2$ may be due to spin alignment effects addressed in Sect. 7.2. The deadzones of the alignment contributions occur at different field orientations than those of the orientation contributions and may thus become more prominent when the (otherwise dominant) orientation contribution vanishes. The increased scatter of the data points near these zones is obviously a consequence of the lower signal/noise ratio near these zones.

In the next step, we fit the $R$-signals by the function

$R_{\mathrm{fit}}\left(\omega_{\mathrm{rf}} ; \omega_{\mathrm{fit}}, \widetilde{\Omega}_{\mathrm{fit}}, A_{\mathrm{fit}}\right)=\frac{A_{\mathrm{fit}} \widetilde{\Omega}_{\mathrm{fit}} \sqrt{\gamma_{\mathrm{exp}}^{2}+\left(\omega_{\mathrm{rf}}-\omega_{\mathrm{fit}}\right)^{2}}}{\left(\omega_{\mathrm{rf}}-\omega_{\mathrm{fit}}\right)^{2}+\gamma_{\mathrm{exp}}^{2}+\widetilde{\Omega}_{\mathrm{fit}}^{2}}$,

where we have fixed the linewidths $\gamma_{\exp }$ to the values $\gamma_{\text {fit }}$ inferred from the phase fits, but leave the resonance frequencies $\omega_{\text {fit }}$ again as free parameters. We note that these fits yield resonance frequency values $\omega_{\mathrm{fit}}$ that agree with those of the phase fits. Those fits also yield the scale factors $A_{\text {fit }}$ and the effective Rabi frequencies $\widetilde{\Omega}_{\text {fit }} . A_{\text {fit }}$ is related to the theoretical amplitude $\widetilde{P}$ in Eq. (9) by $A_{\text {fit }}=\eta \widetilde{P}$, where $\eta$ is a calibration factor (measured in $\mathrm{V} / \mathrm{W}$ ) that converts laser power levels to recorded voltages.

We have performed the above analysis for all field orientations $\left(\theta_{B}, \phi_{B}\right)$, obtaining a set of on-resonance $R$-signal values that can be expressed by the fitted parameters via

$R_{\text {fit }}^{(0)}\left(\theta_{B}, \phi_{B}\right) \equiv R_{\mathrm{fit}}(\delta \omega=0)=A_{\mathrm{fit}} \frac{\gamma_{\mathrm{fit}} \widetilde{\Omega}_{\mathrm{fit}}}{\gamma_{\mathrm{fit}}^{2}+\widetilde{\Omega}_{\mathrm{fit}}^{2}}$,

where $\gamma_{\text {fit }}$ results from the fit by Eq. (16) and $\widetilde{\Omega}_{\text {fit }}$ and $A_{\text {fit }}$ from the fit by Eq. (17). We recall that those equations are universal fit functions that can be applied to $M_{x}$-magnetometer geometries with any orientation of the rf-field.

\section{Results}

The fit of the magnetic resonance spectrum at each field orientation point $\left(\theta_{B}, \phi_{B}\right)$ yields the fit parameters $\omega_{\mathrm{fit}}, \gamma_{\mathrm{fit}}$, $\varphi_{\text {fit }}^{(0)}, \widetilde{\Omega}_{\mathrm{fit}}, A_{\mathrm{fit}}$ as well as the inferred on-resonance signal amplitude $R_{\text {fit }}^{(0)}$ [defined by Eq. (18)] for each grid point. This allows graphical representations of the $\hat{\mathbf{B}}_{0}$ orientation dependencies of all parameters, the orientation dependence of the applied field modulus having already been shown in Fig. (4).

We complement the latter dependence by showing in Fig7a, $b$ the dependencies of the fitted effective Rabi frequencies on the orientation of the field $\mathbf{B}_{0}$ in the $\mathbf{B}_{1} \| \mathbf{k}$ and
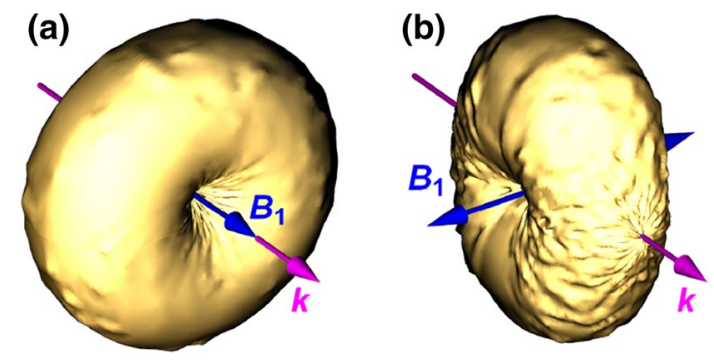

Fig. 7 Measured angular dependencies of the effective Rabi frequencies $\widetilde{\Omega}_{\text {fit }}$ for the $\mathbf{B}_{1} \| \mathbf{k}(\mathbf{a})$ and $\mathbf{B}_{1} \perp \mathbf{k}(\mathbf{b})$ geometries. The surface plots have been obtained by numerical interpolation of $\sim 2400$ discrete data points (some outliers near the dead-zones have been removed). The results are to be compared with the anticipated dependencies shown in Fig. 3
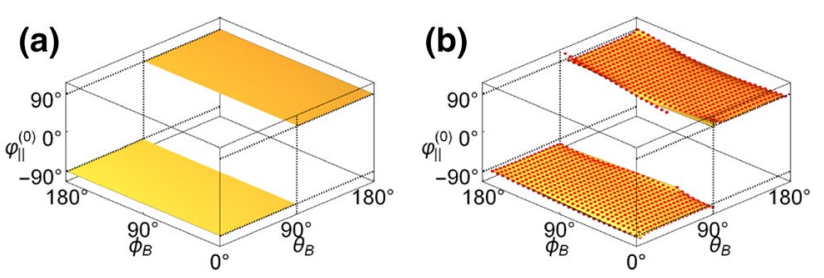

Fig. $8 \mathbf{B}_{1} \| \mathbf{k}$ geometry: Anticipated (a) and measured (b) $\theta_{B}-\phi_{B}$ dependencies of the on-resonance phases $\varphi_{\|}^{(0)}$. The surfaces in the right graph have been obtained by an interpolation algorithm using the discrete data points (shown as red dots)
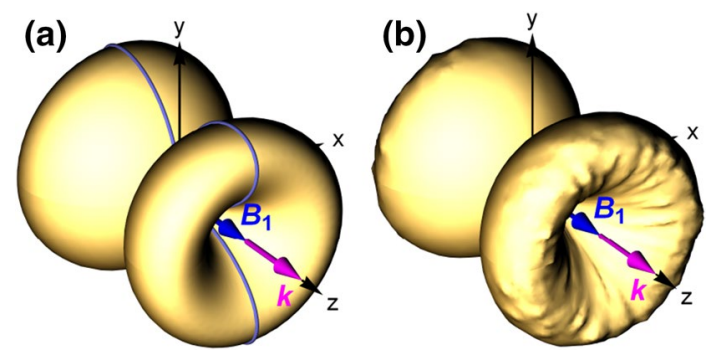

Fig. $9 \mathbf{B}_{1} \| \mathbf{k}$ geometry: Theoretical (a) and experimental (b) angular dependencies of the fitted on-resonance $R$-signals $R_{\text {fit }}^{(0)}$ for $\Omega \approx 2 \gamma$. The solid line on the theoretical graph represents the cut leading to the polar plots shown in Fig. 10

$\mathbf{B}_{1} \perp \mathbf{k}$ geometries. Note that in the plot we have excluded some points near the dead-zones occurring for $\theta_{B}=0^{\circ}$, $90^{\circ}$, and $180^{\circ}$ for which the signals vanish in the noise. The results reflect very well the anticipated dependencies shown in Fig. 3.

In view of magnetometric applications, the resonance amplitudes $R_{\text {fit }}^{(0)}$ and the observed linewidths $\gamma_{\text {fit }}$ are the quantities of main interest, since they determine the magnetometric sensitivity as discussed in Sect. 7.4. While the angular dependence of the relaxation rates has already been 
shown in Fig. 6, we will focus in the following subsections on the angular distributions of the $R_{\mathrm{fit}}^{(0)}$ signals, treating separately the $\mathbf{B}_{1} \| \mathbf{k}$ and $\mathbf{B}_{1} \perp \mathbf{k}$ geometries.

\section{1 $\mathrm{B}_{1} \| \mathbf{k}$ geometry}

We first address the orientation dependence of the on-resonance phase $\varphi_{\|}^{(0)}$. Equation (12) predicts that the $\varphi_{\|}^{(0)}$ can only assume the values $-\pi / 2$ (for $\theta_{B}<\pi / 2$ ) and $+\pi / 2$ (for $\left.\theta_{B}>\pi / 2\right)$ in the $\mathbf{B}_{1} \| \mathbf{k}$ geometry. The experimental findings shown in Fig. 8 reflect very well this anticipated behavior.

In Fig. 9a, b we compare the theoretical prediction and the experimental findings for the $R$-signal in the $\mathbf{B}_{1} \| \mathbf{k}$ geometry. The plots reflect well the rotational symmetry around the k-direction, and show-at least on the displayed scale - a good qualitative agreement.

In order to explore the agreement in a more quantitative manner, we have performed scans of the $\theta_{B}$ angle along the trajectory shown in Fig. 9a at a fixed value of $\phi_{B}$. The results are shown in Fig. 10. Since the anticipated patterns of $R_{\text {fit }}^{(0)}\left(\theta_{B}\right)$ depend on the rf saturation parameter $G_{\text {rf }}=\Omega^{2} / \gamma^{2}$, we have measured these dependencies for different settings of the Rabi frequency $\Omega$, ranging from below-saturation $(\Omega<\gamma)$ to above-saturation $(\Omega>\gamma)$ values. The experimental results are in excellent agreement with theory.

Equations 11 and 15 show that for $\Omega \ll \gamma$ the anticipated $\theta_{B}$ dependence of the $R$-signal is given by $P_{R}^{\hat{z}}(0) \propto\left|\sin ^{2} \theta_{B} \cos \theta_{B}\right|$, a distribution which has a maximum value at $\theta_{B} \sim 54.7^{\circ}$. This particular orientation is indicated by dashed lines in the figure. The data points reflect well this behavior. When the Rabi frequency is increased to saturating values $\Omega \geq \gamma$, the angular distribution becomes more stretched along the $\hat{\mathbf{k}}$ direction.

\section{$6.2 \mathrm{~B}_{1} \perp$ k geometry}

We have performed the same type of measurements in the $\mathbf{B}_{1} \perp \mathbf{k}$ geometry. The magnetometer geometry, determined by the orientations $\hat{\mathbf{k}}$ and $\hat{\mathbf{B}}_{1}$, has no longer a rotational symmetry axis, and one expects this broken symmetry to be reflected in the orientational dependencies of the magnetometer signals. The latter obey a reflection symmetry with respect to the plane determined by $\hat{\mathbf{k}}$ and $\hat{\mathbf{B}}_{1}$.

We start again by addressing first the on-resonance phase, which — because of the broken symmetry-has a non-trivial angular distribution (see Fig. 11). Disregarding some outliers near the dead-zones, which result from large fit errors, the experimental data reflect well the anticipated orientational dependence given by Eq. (14).

The angular dependence of the on-resonance $R$-signal shown in Fig. 12 exhibits well the broken symmetry
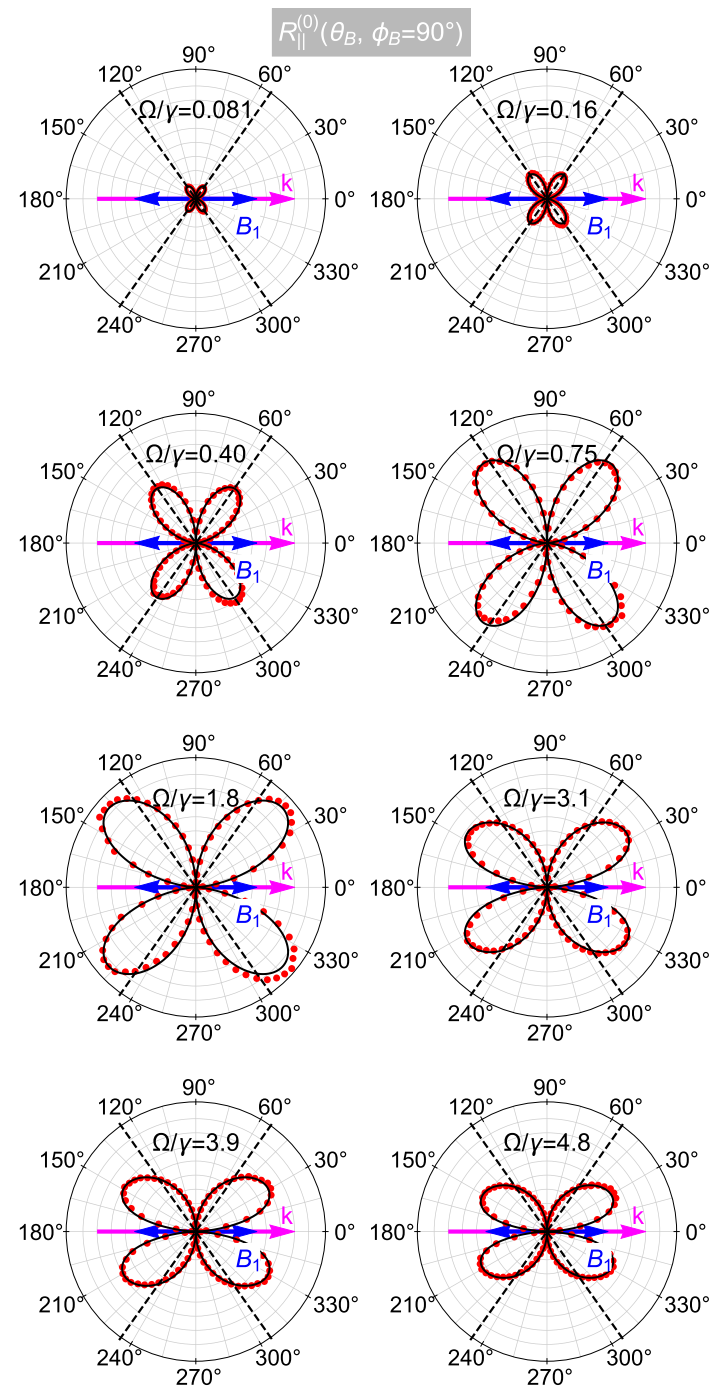

Fig. $10 \mathbf{B}_{1} \| \mathbf{k}$ geometry: Cuts through the angular distributions of Fig. $9 \mathrm{~b}$ for $\phi_{B}=90^{\circ}\left(y-z\right.$ plane). The polar angle $\theta_{B}$ is measured with respect to the $\mathbf{k}$ axis. The Rabi frequency (expressed in units of $\gamma$ ) is varied from below- to above-saturation values. The red dots are data points and the solid (black) lines represent fits with the model function of Eq. (15). The dashed lines represent the orientation $\theta_{B}$ at which the low-saturation data are expected to show a maximum signal

leading to a 'squeezing/stretching' along the direction $\hat{\mathbf{k}} \times \hat{\mathbf{B}}_{1}$. In order to illustrate the broken $\phi_{B}$ symmetry, we have performed angular scans in which we have varied $\phi_{B}$ at a fixed angle $\theta_{B} \sim 45^{\circ}$. The results displayed in Fig. 13a show how the squeezing along $\hat{\mathbf{B}}_{1}$ evolves into a stretching along that direction as $\Omega$ is increased from below- to above-saturation values.

We also show in Fig. $13 \mathrm{~b}, \theta_{B}$-scans in the plane $\phi_{B}=0$, defined by $\hat{\mathbf{k}}$ and $\hat{\mathbf{B}}_{1}$ and in Fig. 13c $\theta_{B^{-}}$scans in the plane $\phi_{B}=\pi / 2$, defined by $\hat{\mathbf{k}}$ and $\hat{\mathbf{k}} \times \hat{\mathbf{B}}_{1}$. Data points are shown together with theoretical fits (solid lines). 

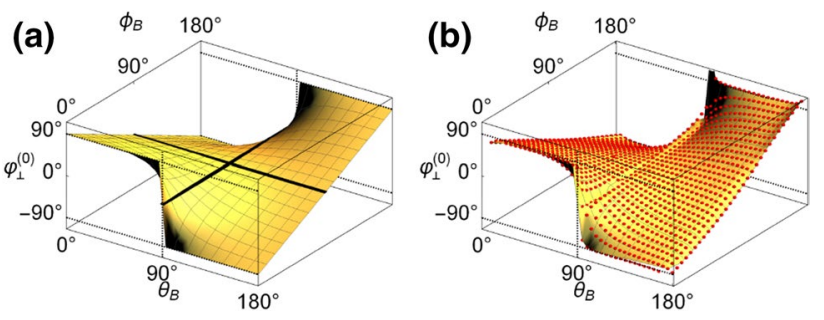

Fig. $11 \mathbf{B}_{1} \perp \mathbf{k}$ geometry: Anticipated (a) and measured (b) $\theta_{B}-\phi_{B}$ dependencies of the on-resonance phases $\varphi_{\perp}^{(0)}$. The surfaces in the right graph have been obtained by an interpolation algorithm using the discrete data points, shown as red dots. The black lines on the left graph indicate $\mathbf{B}_{0}$ orientations for which $\varphi_{\perp}^{(0)}=0$. The red dots on the right graph are data points
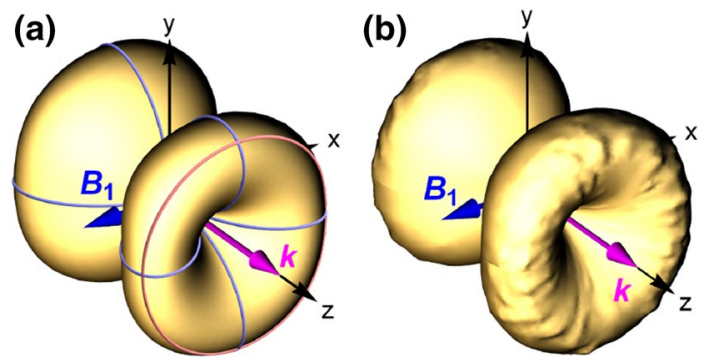

Fig. $12 \mathbf{B}_{1} \perp \mathbf{k}$ geometry: Theoretical (a) and experimental (b) angular dependence of the on-resonance $R$-signals $R_{\text {fit }}^{(0)}$ for $\Omega \approx \gamma$. The reddish and bluish solid lines on the left graph represent the trajectories of the polar plots shown in Fig. 13

\section{Discussion}

We have investigated in detail the dependence of lockin detected signals produced by spin-orientation (vector magnetization)-based $M_{x}$-magnetometers on the orientation of the applied magnetic field of interest $\mathbf{B}_{0}$ and the orientation of the rf-field $\mathbf{B}_{1}$ used to drive the magnetic resonance in the magnetometer. The lock-in signals are fully described by the amplitude ( $R$-signal) and the phase $\varphi$ of the photocurrent oscillation. In all investigated geometries, we find a very good agreement between theoretical predictions and our observations. We can thus claim a successful verification of the general theoretical expressions for $M_{x}$ -magnetometer signals that have been presented recently [3].

\subsection{Experimental asymmetries}

We wish to state nonetheless that achieving the excellent quality of the presented experimental results has been a non-trivial task. One of our goals was to clearly demonstrate the anisotropic angular dependence in the two investigated geometries, viz., the $\mathbf{B}_{1} \| \mathbf{k}$ case (rotational symmetry with respect to $\hat{\mathbf{k}}$, and reflection symmetry with respect to the plane perpendicular to $\hat{\mathbf{k}}$ ), compared to the $\mathbf{B}_{1} \perp \mathbf{k}$ case which lacks the rotational symmetry while keeping the reflection symmetry. In the former case with rotational symmetry, satisfactory results have only been obtained after ensuring that the experimental setup contained no symmetry-breaking elements. On our way to the final results, we have identified and successively eliminated the following perturbing effects:

- Even a tiny elliptical polarization of the light beam leads to the creation of transverse alignment (tensor polarization) in the atomic medium that manifests itself in an anisotropic angular distribution. Special care has therefore been taken to ensure the highest possible degree of circular polarization. For the experiments reported in the paper, the light beam (prior to entering the cell) has a degree of linear polarization (DOLP) of $0.70 \%$, which corresponds to a degree of circular polarization (DOCP) of $>99.99 \%$. In an earlier experiment, we had observed a pronounced anisotropy and discovered later that the light entering the cell in that experiment has had a DOLP of $6 \%$, which corresponded to a DOCP of $99.8 \%$. We note that the DOCP value is given by the corresponding Stokes parameter and that $\mathrm{DOCP}^{2}+\mathrm{DOLP}^{2}=1$ assuming $100 \%$ polarized light. Stress-induced birefringence of the spherical glass cell may slightly alter the light polarization inside of the cell. Any spurious contamination by linear polarization will lead to the creation of transverse spin alignment (tensor polarization), see Sect. 7.2.

- Any magnetic component placed near the Cs vapor cell will perturb the angular distributions in a similar way. We have been able to eliminate such effects by a careful choice and screening of all deployed components for magnetic contaminants.

- In an early stage we used rf-coils of rectangular shape $\left(38 \times 29 \mathrm{~mm}^{2}\right.$, compared to the vapor cell diameter of $\sim 30 \mathrm{~mm}$ ), which led to a pronounced breaking of the rotational $\phi_{B}$-symmetry in the $\mathbf{B}_{1} \| \mathbf{k}$ geometry. Replacing those coils by $100 \mathrm{~mm}$ diameter circular coils has led us to the presented results.

\subsection{Alignment effects}

Despite the taken precautions, some minor imperfections remain in the data:

(a) A slight $(<1 \%)$ anisotropy of the measured Larmor frequencies (Fig. 4b). The maximum quadratic Zeeman shift in the used field of $10 \mu \mathrm{T}$ is $\ll \mathrm{nT}$ and can thus 
be ruled out as cause of the observed deviations at the level of $\lesssim 100 \mathrm{nT}$.

(b) A slight asymmetry of all recorded $R$-signals under saturating conditions $\Omega \geq \gamma$ (Fig. 5a).

(c) A slight anisotropy of the relaxation rates $\gamma$ (Fig. 6b).

(d) A slight forward-backward (with respect to $\mathbf{k}$ ) asymmetry of the $\theta_{B}$ scans in the $\mathbf{B}_{1} \| \mathbf{k}$ geometry (Fig. 10).

The effect (a) may mainly be assigned to field-dependent calibration constants as discussed in Sect. 4. We believe that the origin of the other effects relies in the following imperfection of the magnetometer model [3]: The model assumes that the atomic medium carries only vector spin polarization, commonly referred to as 'orientation,' which, in the language of atomic multipole moments represents a $K=1$ multipole. It is well known [1] that optical pumping with circularly polarized light produces multipole moments $m_{K, Q}$ of ranks $K=1,2, \ldots, 2 F \quad(2 F=8$ in the case of the $F=4$ ground state investigated here), while optical pumping with linearly polarized light produces only even multipole moments $K=2,4, \ldots, 2 F$. It is also known that light interacting with the atoms via an electric dipole transition is only sensitive to the rank $K=1$ and 2 multipole moments [4].

In view of this, pumping with light of perfect circular polarization will not only produce a longitudinal (with respect to $\hat{\mathbf{k}})$ vector polarization $\left(m_{1,0}\right)$, but also a longitudinal alignment (second rank tensor moment $m_{2,0}$ ), whose contribution has magnetic resonance line shapes and angular distributions that differ from the orientation contributions discussed in the paper. By dropping the last term in Eq. (3), we have explicitly ignored alignment contributions. To our knowledge, the effect of the ignored alignment contribution on the $M_{x}$ signals has not been discussed in the literature, despite that the $M_{x}$-magnetometer has been known for more than half a century. One of the reasons for this may be the fact that for many decades $M_{x}$-magnetometers have been operated with discharge lamp light sources, whose broad spectrum does not allow resolving the atomic hyperfine structure, making lamp pumped magnetometers insensitive to alignment contributions in $J=1 / 2$ atoms, such as the alkalis. We recall that alignment contributions have already been observed in [14] and that they are particularly small on the 4-3 hyperfine components of the $D_{1}$ line used here. We are in process of developing a theoretical model allowing us to study this effect in more detail in the near future.

Moreover, the transverse alignment produced by imperfect circular light polarization is also not included in our model.

\subsection{Self-oscillating magnetometer}

We note that the $\mathbf{B}_{1} \perp \mathbf{k}$ geometry is interesting for realizing a so-called self-oscillating magnetometer [2], in which the AC part of the detected photocurrent is used to drive (after suitable amplification and phase shifting) the rf coil. While broadband amplifiers are easy to implement, a broadband phase shifter with fixed amplification is not. We recall that in the $\mathbf{B}_{1} \| \mathbf{k}$ geometry the phase shift $\varphi$ between the photocurrent and the rf-field is $\pm 90^{\circ}$ (Fig. 8), so that a self-oscillating magnetometer in that configuration will always need a phase shifter.

In the $\mathbf{B}_{1} \perp \mathbf{k}$ geometry, on the other hand, the data of Fig. 11 show that arbitrary phase shifts $\varphi$-among them $\varphi=0$ — can be achieved. In that geometry, a suitable orientation of $\mathbf{B}_{0}$ can therefore be used to ensure self-oscillation without the need of a phase shifter. From Fig. 11, one sees that the phase shift $\varphi$ vanishes for fields $\mathbf{B}_{0}$ lying on the circle $\phi_{B}=90^{\circ}$. The $\theta_{B}$ orientation on that circle yielding the maximum $R$-signal depends on the Rabi frequency $\Omega$ as illustrated by the graphs in Fig. 13b and shall be chosen appropriately in order to maximize the signal/noise ratio.

One should note, however, that the $\mathbf{B}_{1} \| \mathbf{k}$ geometry, in which the phase is basically independent of the field orientation is more reliable in terms systematic readout errors: Changing the orientation of the field will not change the phase and will hence not affect the oscillation frequency. The $\mathbf{B}_{1} \perp \mathbf{k}$ geometry, on the other hand, offers the possibility of zero-phase shift operation. However, because of the strongly curved phase vs. orientation surface (Fig. 11), any tilt of the field will change the phase and hence the oscillation frequency. The zero-phase advantage can therefore be brought to full profit only in experiments, in which the direction of $\mathbf{B}_{0}$ does not change.

\subsection{Magnetometric sensitivity}

We end the discussion by addressing the magnetometric sensitivity (or noise-equivalent magnetic field) $\delta B_{\mathrm{NEM}}$ of the $M_{x}$-magnetometer, which can be expressed as

$\left.\delta B_{\mathrm{NEM}} \equiv \frac{1}{\gamma_{\mathrm{F}}} \frac{1}{\mathrm{SNR}}\left|\frac{\mathrm{d} \varphi}{\mathrm{d} \delta \omega}\right|^{-1}\right|_{\delta \omega=0}=\frac{\gamma}{\gamma_{\mathrm{F}}} \frac{\delta R}{R^{(0)}} \propto \frac{\gamma}{R^{(0)}}$,

where $\mathrm{SNR}=R^{(0)} / \delta R$ denotes the signal/noise density ratio, $\delta R$ being the spectral noise density of the total detected light power. Since the on-resonance phase slope $\mathrm{d} \varphi / \mathrm{d} \delta \omega=-\gamma^{-1}$ does not depend on the magnetic field orientation (as supported by our data in Fig. 6), the sensitivity's angular dependence varies only with $R^{(0)}$. Obviously, the larger the amplitude, the higher will be the magnetometric sensitivity, i.e., the smaller will be the detectable field changes.

From Eq. (15), we conclude that the best magnetometric sensitivity is achieved for $\theta_{B}=45^{\circ}$ when the value of $\left|\sin \theta_{B} \cos \theta_{B}\right|$ is maximal for both $\mathbf{B}_{1} \| \mathbf{k}$ and $\mathbf{B}_{1} \perp \mathbf{k}$ case. This argument uses the fact that by adjusting $\Omega$ one can always ensure that the expression 
(a)
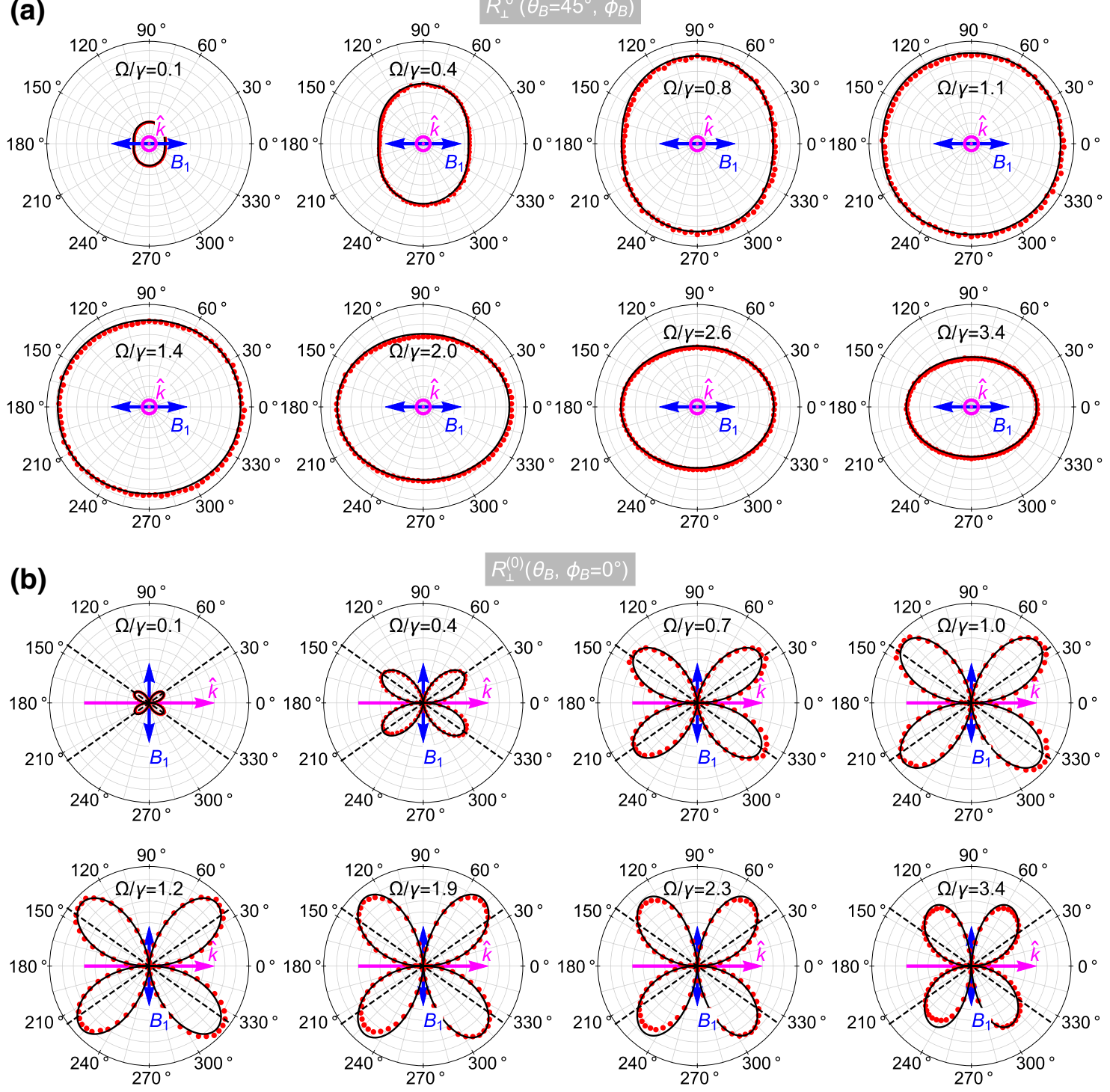

(c)
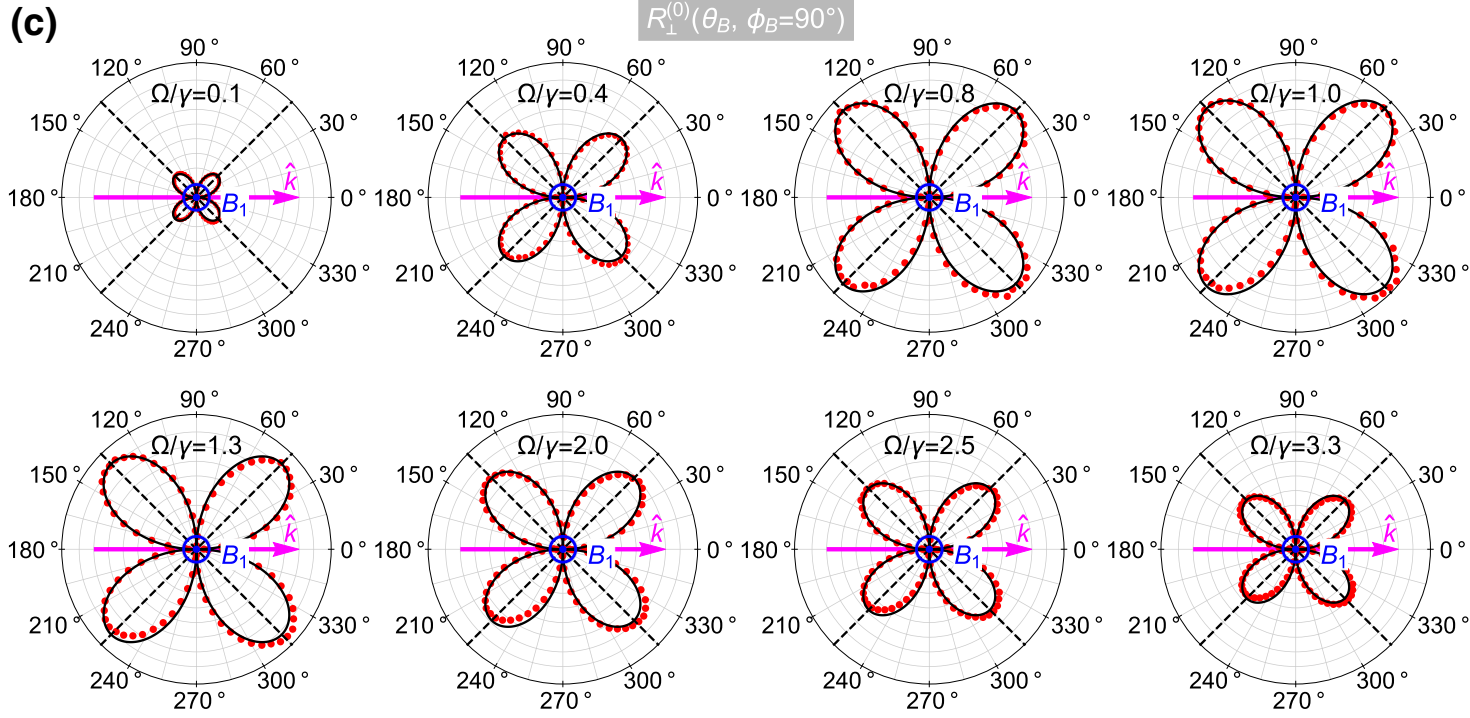
4Fig. $13 B_{1} \perp \mathbf{k}$ geometry: Cuts through the angular distributions of Fig. 12b along the trajectories shown in Fig. 12a. Top graphs (a): $R_{\mathrm{fit}}^{(0)}\left(\phi_{B}\right)$ for $\theta_{B}=\pi / 4$; Middle graphs $(\mathbf{b}): R_{\mathrm{fit}}^{(0)}\left(\theta_{B}\right)$ for $\phi_{B}=\pi / 2$; Bottom graphs $(\mathbf{c}): R_{\mathrm{fit}}^{(0)}\left(\theta_{B}\right)$ for $\phi_{B}=0$. In each group of graphs, the Rabi frequency is varied from below- to above-saturation values. The red dots are data points, and the solid black lines represent fits by the model functions. In graphs $\mathbf{b}$ and $\mathbf{c}$, the dashed lines represent the field orientations yielding maximum signals in the low-saturation limit

$$
\frac{\gamma \widetilde{\Omega}_{\hat{\mathbf{B}}_{1}}\left(\theta_{B}=45^{\circ}, \phi_{B}\right)}{\gamma^{2}+\widetilde{\Omega}_{\hat{\mathbf{B}}_{1}}^{2}\left(\theta_{B}=45^{\circ}, \phi_{B}\right)}
$$

can be made to reach its maximum value of $1 / 2$ for any $\phi_{B}$. Under optimized conditions $\left(\theta_{B}, \phi_{B}, \Omega\right)$ our system has an SNR of $1.5 \times 10^{5} \sqrt{\mathrm{Hz}}$ assuming shotnoise-limited detection of the $4 \mu \mathrm{A}$ DC photocurrent. This yields $\delta B_{\mathrm{NEM}}=13 \mathrm{fT} / \sqrt{\mathrm{Hz}}$, assuming $\gamma / 2 \pi=7 \mathrm{~Hz}$.

\section{Outlook}

We have demonstrated that our algebraic theory of optically detected magnetic resonances is supported with very good accuracy by experimental observations - at least for the two most commonly deployed configurations of the magnetometer. An essential result is the now well-understood interdependent effect of the rf-field strength and static field orientation on the resonance amplitudes and phases. This success encourages us to go one step further and to derive a universal algebraic expression that accounts both for an arbitrary orientation $\left(\theta_{B}, \phi_{B}\right)$ of the static field $\mathbf{B}_{0}$ and-this will be new-for an arbitrary orientation $\left(\theta_{\mathrm{rf}}, \phi_{\mathrm{rf}}\right)$ of the rffield $\mathbf{B}_{1}$. Such an expression may be easily adapted to alloptical designs with Bell-Bloom type of pumping [14-16] as well as to two-beam (pump-probe) experiments. Work in this direction is in progress in our laboratory.

The motivation of the study reported here has been the optimization of an $M_{x}$ magnetometer in view of reaching the highest sensitivity for measuring $\mathbf{B}_{0}$ (and/or changes thereof). On the other hand, the $M_{x}$ principle is also applicable to so-called rf-magnetometry. In that case, a static field $\mathbf{B}_{0}$ of known strength and variable direction is applied to the sensor, and the task is to measure the amplitude and orientation of an unknown rf-field oscillating at a known (or eventually unknown) frequency. $\hat{\mathbf{B}}_{0}$ orientation scans - as the ones deployed here-can then be used to infer the properties of the rf-field. We have performed preliminary investigations along this direction in the frame of our ongoing application of atomic magnetometry to the detection of the magnetic response of small $(\lesssim \mu \mathrm{g})$ samples of magnetic nanoparticles (MNPs). A problem encountered in such experiments is the detection (in view of minimization) of the stray field from a coil system used to harmonically excite the MNP sample [17, 18]. Details of this research shall be published elsewhere.

Acknowledgements We acknowledge financial support by the Physics Department and the Pool de Recherche of the University of Fribourg as well as by Grant No. 200020_162988 of the Swiss National Science Foundation.

Open Access This article is distributed under the terms of the Creative Commons Attribution 4.0 International License (http://creativecommons.org/licenses/by/4.0/), which permits unrestricted use, distribution, and reproduction in any medium, provided you give appropriate credit to the original author(s) and the source, provide a link to the Creative Commons license, and indicate if changes were made.

\section{References}

1. D. Budker, W. Gawlik, D.F. Kimball, S.M. Rochester, V.V. Yashchuk, A. Weis, Rev. Mod. Phys. 74, 1153 (2002)

2. D. Budker, D.F. Jackson Kimball, Optical magnetometry (Cambridge University Press, Cambridge, 2013)

3. A. Weis, G. Bison, Z.D. Grujić, in High Sensitivity Magnetometers, ed. by A. Grosz, M.J. Haji-Sheikh, S.C. Mukhopadhyay Springer, Berlin (2017)

4. W. Happer, Rev. Mod. Phys. 44, 169 (1972)

5. A.L. Bloom, Appl. Opt. 1, 61 (1962)

6. A. Ben-Kish, M.V. Romalis, Phys. Rev. Lett. 105, 193601 (2010)

7. T. Wu, X. Peng, Z. Lin, H. Guo, Rev. Sci. Instr. 86, 103105 (2015)

8. C. Hovde, B. Patton, O. Versolato, E. Corsini, S. Rochester, D. Budker, Heading error in an alignment-based magnetometer, Proc. SPIE 8046, 80460Q (2011)

9. J. Kitching, S. Knappe, V. Shah, P. Schwindt, C. Griffith, R. Jimenez, J. Preusser, L.-A. Liew, J. Moreland, in IEEE International Frequency Control Symposium (2008), pp. 789-794

10. E. Breschi, Z.D. Grujić, P. Knowles, A. Weis, Appl. Phys. Lett. 104(2), 023501 (2014)

11. N. Castagna, G. Bison, G. Di Domenico, A. Hofer, P. Knowles, C. Macchione, H. Saudan, A. Weis, Appl. Phys. B 96(4), 763 (2009)

12. N. Castagna, A. Weis, Phys. Rev. A 84, 053421 (2011)

13. E. Breschi, Z.D. Gruijć, A. Weis, Appl. Phys. B 115, 85 (2014)

14. Z.D. Grujić, A. Weis, Phys. Rev. A 88, 012508 (2013)

15. I. Fescenko, P. Knowles, A. Weis, E. Breschi, Opt. Express 21(13), 15121 (2013)

16. E. Breschi, Z.D. Gruijć, P. Knowles, A. Weis, Phys. Rev. A 88, 022506 (2013)

17. S. Colombo, V. Lebedev, Z.D. Grujić, V. Dolgovskiy, A. Weis, Int. J. Magn. Part. Imaging 2, 1606002 (2016)

18. S. Colombo, V. Lebedev, Z.D. Grujić, V. Dolgovskiy, A. Weis, Int. J. Magn. Part. Imaging 2, 1604001 (2016) 